\title{
金属银膜生长过程中的分形现象 及动力学模型
}

骆柱蓬 艾竹茖 韦 铅

(东南大学吴健雄实验室,南京 210018)

\section{关链调电化学沉积、单分子膜、分形、动力学}

\section{1 引言}

有关用电化学沉积方法进行金属凝聚体生长规律的研究在过去的十年中取得了很大进 展 ${ }^{[1-9]}$. 这应归功于 Witten 和 Sander 在 1981 年提出的受限扩散凝聚 (DLA) 模型 ${ }^{[1]}$. 将这 一模型成功地用以解释二维空间金属电化学沉积过程及其形态的早期实验是日本的 Matsushita 等在 1984 年完成的 ${ }^{[2]}$. 此后, 对这一凝聚态物理中的重要领域的研究不断深入细致, 从 生长条件(如: 溶液浓度, 电场强度)的变化 ${ }^{[3-6]}$; 到生长空间的改变 (如: 圆形、矩形等) ${ }^{[n}$; 从 简单的维数计算 ${ }^{[2]}$ 到后来的多标度分形动力学模型等 ${ }^{[8,9]}$, 都有了不少突 破. 1990 年 Zhao 等 ${ }^{[10]}$ 人将电解液表面铺上单分子膜, 用电化学还原方法, 在恒电位情况下, 生长出二维超微粒 银獏, 并测试和分析了该类膜的固态特性, 但并未报道有关分形的现象. 早在 Matsushita 等 人的实验中也用了正丁基醋酯作为溶液的分界面, 但这与用单分子层作为界面有本质不同. 随 后的金属凝聚体生长实验多用极薄的三明治结构来近似二维空间. 本文报告的内容是上述两 个研究方向的交叉点, 即在液面单分子层诱导下用恒电流控制生长所凝聚的银膜具有两种宏 观形态: 封闭生长的二维致密凝聚内部和开放生长的扩散分形边界. 其临界点正是由溶液电 解电压决定的. 对此我们分析了其生长机理并建立了一动力学模型. 将这一模型结果与实验 结果比较,两者在形态和边界维数上是相当一致的.

\section{2 实验及结果}

实验装置如图 1 所示. 直径为 $9 \mathrm{~cm}$ 高为 $1 \mathrm{~cm}$ 的圆形玻璃容 器 内装 $\mathrm{AgNO}_{3}$ 溶 液 3$4 \mathrm{~mm}$. 阴极是直径为 $0.1 \mathrm{~mm}$ 的铂丝, 置于容器中心正上方. 阳极则用金属银片制成的圆环. 整个容器放在体视显微镜 (XTL-1 型)下进行动态观察并可接照相及摄象系统. 界面材料选 用花生酸和聚合物液晶两种. $\mathrm{AgNO}_{3}$ 溶液浓度为 $0.001 \mathrm{~mol}$, 用去离子水配制. 在铺单分子膜 前, 用抽水洜将液面灰尘吸去, 然后用微量注射器缓慢将约 $10 \mu 1$ 的花生酸(或液晶)溶液(它们 均溶解于氯仿之中, 浓度为 $0.001 \mathrm{~mol}$ ) 沿液面滴人容器中. 阴极触膜须用微调下降装制, 该下 降装置是手摇式机械传动垂直运动的机构, 阴极铂丝沿坚直方向固定在装置的运动头上. 采 用恒电位和恒电流两种方式进行生长 ( HDV - 7C 晶体管恒电位仪). 下面给出一些典型实 验结果. 


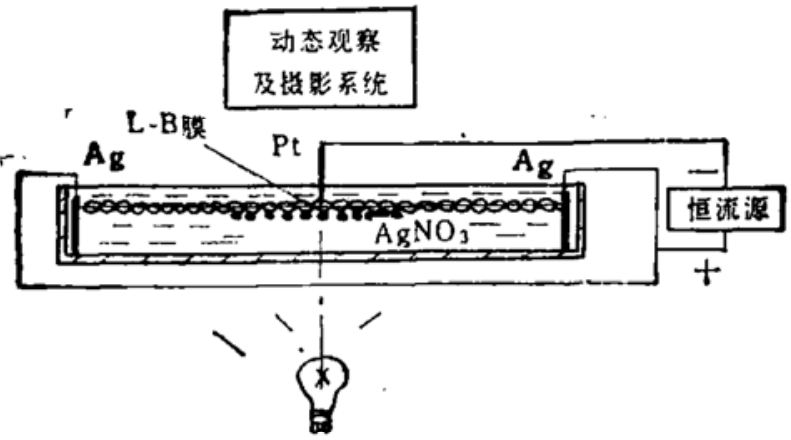

图 1 恒电流条件下电化学沉积银膜的实验装置简图

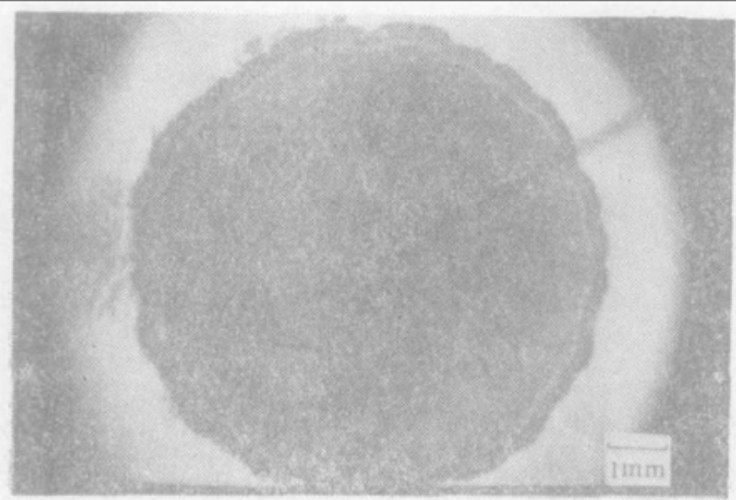

（s）佰电流条件下生长出的银膜结构

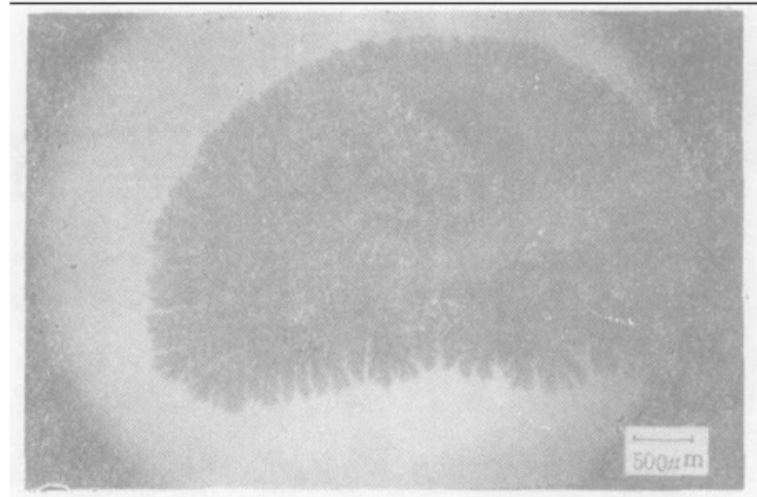

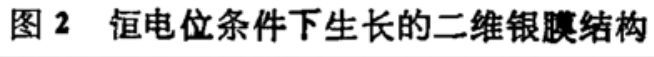

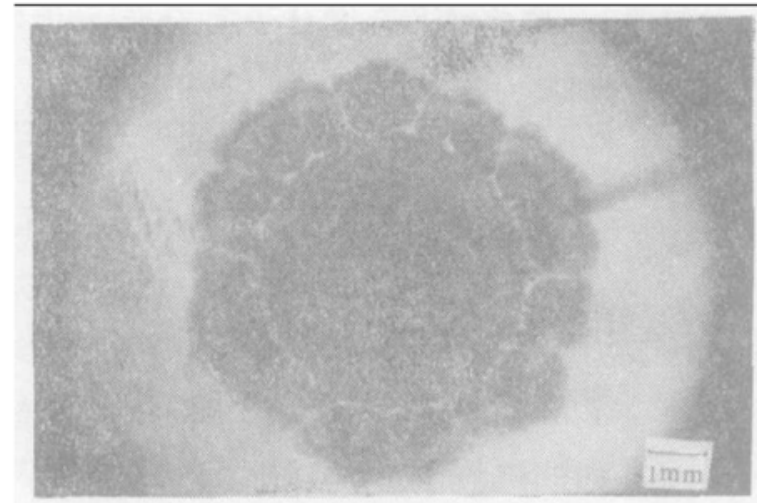

(b) 生长结构随电流变化的结果

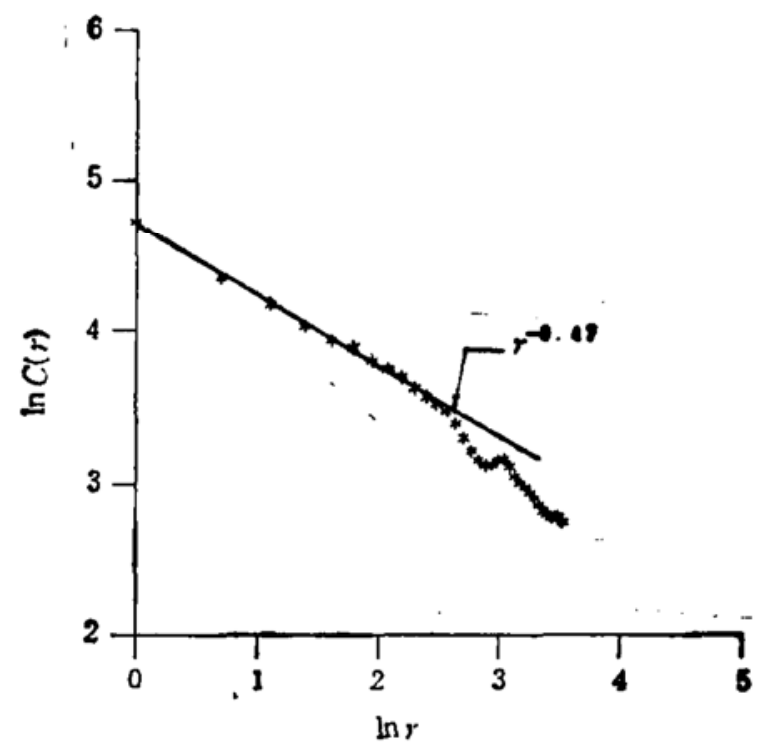

(c) 银膜边界相变的分形结构

（d）（c）图中局部密度相关函数 $c(r)$ 和标尺r 的 双对数曲线 $D=1.52 \pm 0.02$

图 3

为进行比较, 我们先进行了恒电位条件下二维银膜的生长, $\mathrm{AgNO}_{3}$ 浓度为 $0.001 \mathrm{~mol}$, 液 面单分子层材料是花生酸, 恒电位为 $0.82 \mathrm{~V}$. 从图 2 给出的生长形态看, 与文献 [10] 的结果 一致,它并不具有明显的宏观分形结构, 银膜沿生长平面较为均匀, 只是生长速度越来越慢, 部 分边界开始进行优势生长. 为观察到更丰富的生长形态, 我们采用恒电流控制方法, 液面单分 子层材料用聚合物液晶. 图 3(a) 是在 $I=0.131 \mathrm{~mA}$ 时 $5 \mathrm{~min}$ 内生长的二维银膜形态, 随后 生长速度渐慢, 溶液中电场电位缓慢下降, 边界处可观察到一些小的枝状晶体, 将电流增大到 
$0.151 \mathrm{~mA}$, 通过体视显微镜可观察到新的生长从边界处均匀向外成扇形迅速扩大, 此时电位在 相应增加之后再次缓慢下降, 再次提高电流 $I=0.171 \mathrm{~mA}$, 生长情况基本类似, $30 \mathrm{~min}$ 后, 已 可清楚地看到边界的树枝状晶体 (图 3(b)), 通过局部放大 (图 3(c)), 取出边界部分区 域, 用计算机处理, 根据密度相关函数测得其 平均分维数是 $D=1.52 \pm 0.02$ (图 3(d))

\section{3 生长机理分析及动力学模型设计}

在恒电位生长条件下, 开始电流近似线 性增加, 银膜不断长大, 其形态是明显的二维 结构. 但不久开始体内生长, 电流也几乎不 再变化. 改用恒电流控制时, 由于沉积速度 佰定, 电场电压也随生长的进行逐渐下降, 直 至接近临界电压值. 随后的生长便是在这一 临界状态下进行, 此时电场漂移的影响减小, 随机扩散凝聚增强, 从而便形成一个开放的

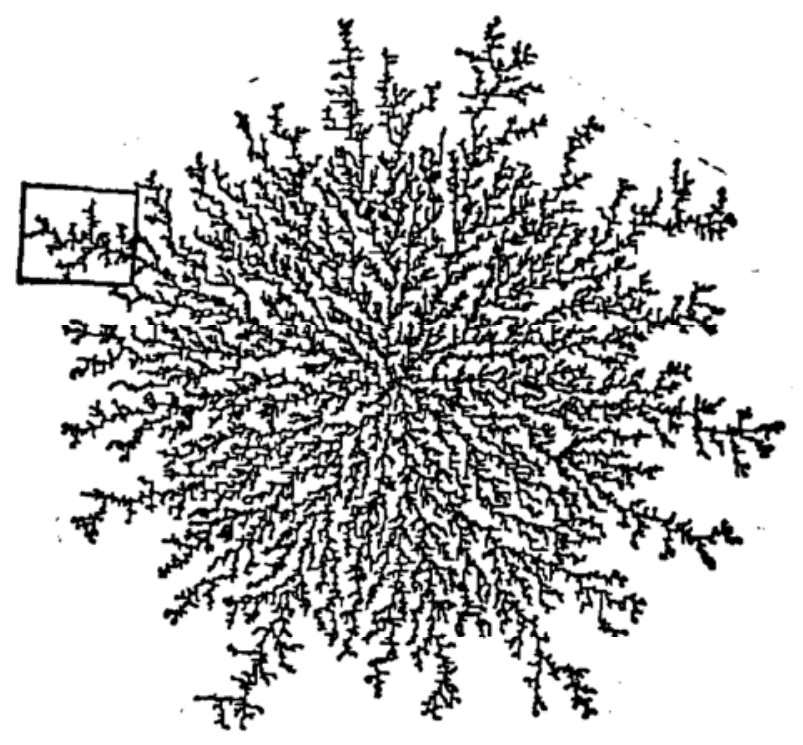

图 4 计算机动力学生长模型结果 图边界处 $D=1.53 \pm 0.01$ 分形树枝状边界. 从图 3(c) 中可明显观察到这一相变结构.

为模拟这一生长的动力学过程, 我们在 DLA 模型基础上, 将随机粒子扩散的几率 $p_{\mathrm{i} j}$ 定 义为: $p_{i j}=1 / 4+k\left(\varphi_{j}-\varphi_{i}\right)$, 式中第一项是 DLA 模型中的随机扩散几率, 第二项是考虑 了电场影响, 使得扩散粒子向阴极中心漂移的几率增加, $k$ 是相应电场强度大小, $\varphi_{\mathrm{i}}, \varphi_{\mathrm{i}}$ 是粒 子指向中心的参数. 当生长到临界状态时 $k \rightarrow 0$. 这时 $p_{\mathrm{ij}}=1 / 4$, 便完全是 DLA 生长了. 模拟过程是在 Silicon 工作站进行 (见图 4), 可以看到其形态和实际生长图形一致. 同样方法 测得其边界处的分维数 $D=1.53 \pm 0.01$, 也和实际图形计算出的结果一致.

\section{4 讨 论}

根据实际生长及模拟结果, 我们认为, 致密两维结构的形成和液面单分子层特性有关, 而 边界分形结构是随机扩散凝聚的结果, 它与液面材料性质无关. 因此, 动力学模型基本揭示了 这类生长的结构和规律. 有关边界处凝聚体的相变行为还需进一步研究.

\section{参考文献}

[1] Witten, T.A., Sander, L.M., Phys. Rev. Lett., 1981, 47 (9):1400.

[2] Matsushita, M. et al., Phys. Rev. Lett., 1984, 53(3):286.

[3] Sawada Yasuji et al., Phys. Rev. Lett., 1986, 56(12):1260.

[4] Grier, D. et al., phys. Rev. Lett., 1986, 56(12):1264.

[5] Hibbert, D.B., Melrose, J.R., Phys. Rev. A, 1988, 38 (2): 1036.

[6] Melrose, J.R., Hibbert, D.B., Phys. Rev. Lett., 1990, 65 (24):3009.

[7] Fleury, V., Rosso, M., Phys. Rev. A, 1991, 43 (12):6908.

[8] Takaishi Nagatani et al.,Phys. Rev. A, 1991, 44 (10): 6723.

[9] fleury, V., Rosso, M., Phys. Rey. Lett., 1992, 68(6):2492.

[10] Zhao, X.K., Fendler, J.H., J. Phys. Chem., 1990, 94(9):3386. 